\title{
Engineered T cell therapies
}

\author{
ANNE-CHRISTINE FIELD*, WASEEM QASIM \\ Molecular Immunology Unit, Institute of Child Health (ICH), University College London (UCL), 30 Guilford \\ Street, London WC1N 1EH, UK
}

\begin{abstract}
Alongside advancements in gene therapy for inherited immune disorders, the need for effective alternative therapeutic options for other conditions has resulted in an expansion in the field of research for $\mathrm{T}$ cell gene therapy. $\mathrm{T}$ cells are easily obtained and can be induced to divide robustly ex vivo, a characteristic that allows them to be highly permissible to viral vector-mediated introduction of transgenes. Pioneering clinical trials targeting cancers and infectious diseases have provided safety and feasibility data and important information about persistence of engineered cells in vivo. Here, we review clinical experiences with $\gamma$-retroviral and lentiviral vectors and consider the potential of integrating transposon-based vectors as well as specific genome editing with designer nucleases in engineered $\mathrm{T}$ cell therapies.
\end{abstract}

\section{Introduction}

Gene therapy involves the introduction of genetic material (including DNA, RNA, small interfering RNA or antisense oligonucleotides) into cells to correct cellular dysfunction or to provide new functions in order to cure or slow the progression of diseases. To date, over 1800 gene therapy clinical trials have been approved worldwide (Ref. 1). T cell gene therapy represents an emerging therapeutic option for use against malignancies, infections and certain monogenic diseases. The ability to harvest, manipulate and reinfuse engineered $\mathrm{T}$ cells has made them attractive targets for gene therapy. Pioneering trials have already been undertaken to treat inherited $\mathrm{T}$ cell deficiency, redirect $\mathrm{T}$ cell antigen specificity, confer resistance to human immunodeficiency virus (HIV) and incorporate suicide genes to safeguard against graft-versus-host disease (GvHD) in the allogeneic transplant setting. As $\mathrm{T}$ cells are long lived cells, with periods of quiescence and active proliferation, integrating vector systems have generally been used to modify $\mathrm{T}$ cells, and here we review clinical experiences with $\gamma$-retroviral and lentiviral vectors ( $\gamma$ RVs and LVs, respectively) and consider the potential of integrating transposon-based vectors, as well as transient expression of gene-modifying nuclease enzymes.

\section{Long-term gene expression in T cells mediated by $\gamma \mathrm{RV}$}

Murine $\gamma \mathrm{RV}$ with intact long-term repeats (LTRs) flanking a transgene expression cassette, and packaged using stable cell lines, which provide accessory packaging and envelope components, have been widely exploited for $\mathrm{T}$ cell modification. Efficient $\gamma \mathrm{RV}$ mediated gene integration requires actively proliferating target cells, and $\mathrm{T}$ cell activation procedures have evolved from PHA stimulation, to activation with
anti-CD3 antibody and currently beads conjugated with anti-CD3 and anti-CD28 antibodies.

$\gamma$-retroviral $\mathrm{T}$ cell gene therapies are summarised in Table 1. Initial gene marking studies (Ref. 2) tracked tumour-infiltrating lymphocytes mediating regression of metastatic melanoma using a retroviral vector encoding a gene for resistance to neomycin. Soon afterward, autologous $\mathrm{T}$ cells from infants with adenosine deaminase (ADA) enzyme deficiency, a form of severe combined immunodeficiency (SCID), were reconstituted using $\gamma \mathrm{RV}$ (Refs 3, 4). Both patients remained free from serious infections, on reduced doses of enzyme replacement (Ref. 5). Although the clinical benefit was limited because of low gene transfer rates, retroviral gene transfer proved feasible, and gene-modified autologous cells persisted long-term without causing adverse effects.

\section{Suicide gene therapies}

Another early $\mathrm{T}$ cell immunotherapy trial recruited patients with HIV infection and modified CD8+ cytotoxic $\mathrm{T}$ cells by retroviral transduction with a herpes simplex virus thymidine kinase (HSV-TK) fusion gene. Although technically feasible, the main limitation was host-mediated immune responses against the transgene (Ref. 6). In 1997, Bonini et al. reported the use of allogeneic $\mathrm{T}$ cells engineered to express HSVTK to render cells sensitive to the prodrug ganciclovir (GCV) and safeguard against GvHD (Ref. 7), and similar studies were subsequently reported by a number of other groups (Refs 8,9). Donor $\mathrm{T}$ cells modified with the HSV-TK suicide gene were administered to patients with leukemic relapse and GvHD was effectively controlled by GCV-induced elimination of the transduced cells (Ref. 7). Tiberghien et al. also demonstrated that the use of HSV-TK expressing donor $\mathrm{T}$ cells in 12 patients at the time of allogeneic 
TABLE 1.

T CELL GENE THERAPY CLINICAL TRIALS.

\begin{tabular}{|c|c|c|c|}
\hline Diseases targeted & $\begin{array}{l}\text { Vectors } \\
\text { designed }\end{array}$ & Strategy implemented & References \\
\hline \multicolumn{4}{|l|}{ Inherited } \\
\hline ADA & $\gamma \mathrm{RV}$ & ADA cDNA gene transfer in autologous PBL cells (and BM) & $(3,4)$ \\
\hline \multicolumn{4}{|c|}{ 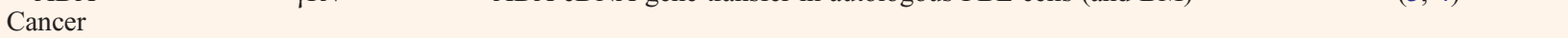 } \\
\hline \multirow[t]{8}{*}{ Leukaemia } & $\gamma \mathrm{RV}$ & $\begin{array}{l}\text { Suicide gene transfer ( } \triangle \text { LNGFR/ HSV-TK, NeoR/HSV-TK) in allogeneic } \\
\text { PBLs }\end{array}$ & $(7,9,10)$ \\
\hline & $\gamma \mathrm{RV}$ & $\begin{array}{l}\text { Suicide gene transfer ( } \triangle \mathrm{LNGFR} / \mathrm{HSV}-\mathrm{TK} \text { ) in HLA-haploidentical donor } \\
\text { PBMCs }\end{array}$ & $(11,12,13)$ \\
\hline & $\gamma \mathrm{RV}$ & Suicide gene transfer $(\triangle \mathrm{CD} 34 / \mathrm{HSV}-\mathrm{TK})$ in haploidentical donor PBMCs & (14) \\
\hline & $\gamma \mathrm{RV}$ & Suicide gene transfer (iCasp9/ $\Delta$ CD19) & (15) \\
\hline & $\gamma \mathrm{RV}$ & Tumour specific CAR gene transfer (CD19) in autologous PBLs & $(29,30,31,32)$ \\
\hline & $\gamma \mathrm{RV}$ & Tumour specific CAR gene transfer (CD19) in allogeneic PBLs & (33) \\
\hline & $\gamma \mathrm{RV}$ & Tumour specific CAR gene transfer (CD19) in allogeneic virus-specific PBLs & (34) \\
\hline & LV & Tumour specific CAR gene transfer (CD19) in autologous PBLs (ALL) & (77) \\
\hline \multirow[t]{2}{*}{ Lymphoma } & LV & Tumour specific CAR gene transfer (CD19) in autologous PBLs & $(73,78)$ \\
\hline & SB & Tumour specific CAR gene transfer (CD19) in autologous PBLs & (93) \\
\hline \multirow[t]{5}{*}{ Melanoma } & $\gamma \mathrm{RV}$ & Selectable marker gene transfer (NeoR) in autologous TILs & \\
\hline & $\gamma \mathrm{RV}$ & $\begin{array}{l}\text { Tumour specific TCR gene transfer (MART-1 and gp-100) in autologous } \\
\text { PBLs }\end{array}$ & $(16,17,18)$ \\
\hline & $\gamma \mathrm{RV}$ & Tumour specific TCR gene transfer (NY-ESO-1) in autologous PBLs & (19) \\
\hline & $\gamma \mathrm{RV}$ & Tumour specific TCR gene transfer (MAGE -A3) in autologous PBLs & $(20)$ \\
\hline & LV & Tumour specific TCR gene transfer (MAGE -A3) in autologous PBLs & (79) \\
\hline Neuroblastoma & $\gamma \mathrm{RV}$ & Tumour specific CAR gene transfer (GD2) in EBV-CTLs & $(26,25)$ \\
\hline Ovarian cancer & $\gamma \mathrm{RV}$ & Tumour specific CAR gene transfer ( $\alpha$ Folate Receptor) in autologous PBLs & (24) \\
\hline $\begin{array}{l}\text { Renal cell } \\
\text { carcinoma }\end{array}$ & $\gamma \mathrm{RV}$ & $\begin{array}{l}\text { Tumour specific CAR gene transfer (Carbonic Anhydrase IX) in autologous } \\
\text { PBLs }\end{array}$ & (23) \\
\hline $\begin{array}{l}\text { Synovial cell } \\
\text { sarcoma }\end{array}$ & $\gamma \mathrm{RV}$ & Tumour specific TCR gene transfer (NY-ESO-1) in autologous PBLs & $(19)$ \\
\hline \multicolumn{4}{|l|}{ Infectious diseases } \\
\hline \multirow[t]{8}{*}{ HIV } & $\gamma \mathrm{RV}$ & Suicide gene transfer (HSVTK) in autologous CD8T cells & (6) \\
\hline & $\gamma \mathrm{RV}$ & Anti-HIV Rev gene transfer in autologous CD4T cells & (36) \\
\hline & $\gamma \mathrm{RV}$ & Anti-HIV ribozyme gene transfer in autologous CD4T cells & (37) \\
\hline & $\gamma \mathrm{RV}$ & Anti-HIV tat ribozyme gene transfer in syngeneic CD4T cells & (40) \\
\hline & $\gamma \mathrm{RV}$ & Anti-HIV Rev gene transfer in syngeneic CD4T cells & (41) \\
\hline & $\gamma \mathrm{RV}$ & HIV-specific chimeric TCR gene transfer (CD4zeta) in autologous PBLs & $(38,39)$ \\
\hline & LV & $\begin{array}{l}\text { Antisense segment targeted against HIV envelope gene (ASenv) transfer in } \\
\text { autologous CD4T cells }\end{array}$ & $(70,76)$ \\
\hline & $\gamma \mathrm{RV}$ & Gp41-derived fusion peptide inhibitor gene transfer in autologous CD4T cells & (42) \\
\hline
\end{tabular}

$\mathrm{BM}$, bone marrow; CAR, chimeric antigen receptors; $\mathrm{CD}$, cluster of differentiation; EBV-CTLs, Epstein-Barr virus cytotoxic $\mathrm{T}$ lymphocytes; GD2, ganglioside 2; HIV, human immunodeficiency virus; HLA, human leukocyte antigen; HSVTK, human herpes simplex virus thymidine kinase; LV, lentiviral-vector; MAGE-A3, melanoma-associated antigen 3; MART, melanoma antigen recognized by T Cells NY-ESO; PBMCs, peripheral blood mononuclear cells; TCR, T cell receptor; TIL, tumour-infiltrating lymphocyte; $\gamma \mathrm{RV}, \gamma$-retroviral vector.

bone marrow transplantation was feasible and associated with persistent circulation of modified cells, GCV-sensitive GvHD, and the absence of acute toxicity (Ref. 9). A similar clinical strategy using an almost identical vector with HSV-TK/NeoR led to secondary graft rejection in two of three treated patients due to immunogenicity of infused T cells (Ref. 10).

In subsequent multicentre trials, leukemic patients were infused with donor lymphocytes expressing the HSV-TK suicide gene, following haploidentical stemcell transplantation. It was reported that 22 of 28 patients who received genetically modified $T$ cells achieved rapid $\mathrm{T}$ cell immune recovery, with the development of a polyclonal repertoire protective against pathogens, leading to a significant long-term reduction of infection-related mortality. Of note, ten patients developed acute GvHD and one developed chronic GvHD, which were controlled by induction of the suicide gene (Ref. 11). In a follow-up study of these 22 patients, new insights into the biologic events leading to the immune reconstitution after HSV-TK cell infusions were provided. It was suggested that the infused $\mathrm{T}$ cells that carried the suicide gene supported de novo generation of $\mathrm{T}$ cells from thymus-dependent pathways, leading to long-term immune reconstitution (Ref. 12). In other studies, transduced $\mathrm{T}$ cells were detectable in nine leukemic patients for up to 5 years with seven of nine patients alive and in complete remission (Ref. 13). A recent publication reports the production and first-in-man use of mismatched donor T cells modified with a $\gamma \mathrm{RV}$ expressing a HSV-TK fused to a truncated CD34 marker protein for magnetic selection. Circulating gene-modified $\mathrm{T}$ cells were detectable by flow cytometry and by molecular tracking in all three subjects. There was resolution of viral infections, concordant with detected antigen-specific $\mathrm{T}$ cell responses, and gene-modified cells persisted for over a year (Ref. 14).

As HSV-TK is virus-derived and immunogenic, alternative suicide genes have also been investigated. Di Stasi et al. devised an inducible T cell safety switch that is based on the human caspase 9. When exposed to a synthetic dimerising drug, the inducible caspase 9 
becomes activated and leads to the rapid death of cells expressing the iCasp9/ $\Delta \mathrm{CD} 19$ construct. Five paediatric patients who had undergone stem cell transplantation for relapsed acute leukaemia were treated with high numbers of genetically modified $\mathrm{T}$ cells. Four patients developed GvHD, which was treated with a single dose of dimerising drug, eliminating over $90 \%$ of the modified $\mathrm{T}$ cells within $30 \mathrm{~min}$ of the administration and ended GvHD without recurrence (Ref. 15).

\section{$\alpha \beta T$ cell receptor (TCR) gene therapies}

Similar retroviral vectors have been used to redirect TCR specificity using $\alpha \beta$ TCRs. Morgan RA et.al. transduced autologous T cells from human leukocyte antigen (HLAA201) melanoma patients with a retroviral vector constructed and optimised to express a MART-1 $\alpha \beta$ TCR. In 17 patients with progressive metastatic melanoma, gene transfer efficiencies ranged from 17 to $67 \%$ in peripheral blood lymphocytes. Overall, two patients $(12 \%)$ demonstrated a sustained regression of their metastatic melanoma, with high levels of circulating engineered cells at 1 year after infusion (Ref. 16). A follow-up study provided a detailed longitudinal analysis of cell persistence and transgene expression in vivo. Interestingly, transgene expression was repressed over time, and silencing was not associated with methylation but rather with transcriptional down-regulation, which was reversible upon lymphocyte reactivation (Ref. 17). In subsequent trials carried out with higher avidity TCRs, objective clinical responses were seen in 30\% of patients $(6 / 20)$ treated with autologous MART-1 TCR modified T cells and in $19 \%$ of patients $(3 / 16)$ treated with a gp100-reactive TCR (Ref. 18). Gene transfer efficiencies represented $\geq 10 \%$ of peripheral blood lymphocytes in all patients 1 month following treatment. Some subjects encountered adverse effects related to ontarget reactivity in normal tissues expressing melanin antigens, including the eye, ear and skin.

Similar approaches have been used in patients with refractory metastatic melanoma or metastatic synovial cell sarcoma with NY-ESO-1-positive tumours (Ref. 19). After approximately 1 month, gene marking ranged between 2 and $60 \%$ of CD8 $+\mathrm{T}$ cells present in peripheral blood (in 14 of the 17 treated patients). Four of six patients with synovial cell sarcoma achieved objective clinical responses, with one lasting 18 months, and five of 11 patients with melanoma also achieved objective clinical responses, including two complete responses on-going at 22 and 20 months and one partial response on-going at 9 months. Morgan RA et al. (Ref. 20) also reported the clinical application of MAGE-A3 TCR gene-modified T cells in an adoptive cell therapy trial in nine cancer patients (seven metastatic melanoma, one synovial sarcoma and one oesophageal cancer). At 1 month post-treatment, TCR positive cells in the circulation were in the range of $11-84 \%$ with a mean of $41 \%$, and five patients experienced clinical regression of their cancers. However, on-target neurologic toxicities were reported in three cases, and two proved fatal (Ref. 20). Autologous lymphocytes engineered to express HLA-A201/HBs183-91 $\alpha \beta$ TCR specific for the hepatitis $B$ virus (HBV) surface antigen (HBsAg) have been used to target HBV-associated hepatocellular carcinoma in a subject with chemoresistant extrahepatic metastatic disease (Ref. 21). Forthcoming trials in London aim to use the same vector platform to target Wilms' tumor antigen 1 (WT1), a tumour associated antigen that may be a useful target in relapsed acute myeloid leukaemia (AML) and other haematological malignancies (ClinicalTrials.gov: NCT01621724, UK-0150), as well as cytomegalovirus, a common pathogen in immunosuppressed transplant patients (EudraCT No: 2008-006649-18, UK-0190).

\section{Chimeric antigen receptor (CAR) gene therapies}

CARs are recombinant receptors that combine the specificity of an antigen-specific antibody with T-cell signalling domains (Fig. 1) and have been recently modified to include co-stimulation domains (Ref. 22). Firstgeneration CARs used in these studies were composed of signalling domains derived from single molecules, either the $\mathrm{CD} 3-\zeta$ chain or $\mathrm{Fc}$ receptor $\gamma(\mathrm{FcR} \gamma)$ chain. Many trials that used gene-modified lymphocytes with first-generation CARs encountered low levels of cell persistence. For example, poor clinical responses were seen in three patients with renal cancer who received autologous T cells transduced with a CAR directed against carbonic anhydrase-IX (Ref. 23) or in eight patients with ovarian cancer who received autologous PBMCs transduced to express a CAR targeting the $\alpha$-folate receptor (Ref. 24). In addition, immunogenicity of CARs was observed, as well as on-target adverse effects in nontumour tissues (Ref. 23). Pule et al. engineered EpsteinBarr virus-specific cytotoxic T lymphocytes (CTLs) to express a CAR directed against diasialoganglioside GD2, a non-viral tumour-associated antigen expressed by human neuroblastoma cells. They reasoned that these genetically engineered lymphocytes would receive optimal costimulation after engagement of their native receptors, enhancing survival and antitumour activity mediated through their chimeric receptors (Ref. 25). Four of the eight patients with evaluable tumours had evidence of tumour necrosis or regression including a complete and sustained remission. In a follow up study, the analysis of the long-term fate of these low-level persisting CAR cells was performed on a total of 19 subjects with high-risk neuroblastoma, including the original 11 patients. CAR cells persisted at low levels for as long as 4 years, with a highly statistically significant association between prolonged detection and the number of helper CD4 and central memory $\mathrm{T}$ cells. Of the 11 patients with active disease at the time of infusion, three patients achieved complete response (Ref. 26).

Second-generation CARs incorporating additional signalling domains derived from costimulatory molecules such as CD28 were assessed against B cell lymphomas expressing the CD19 antigen (Ref. 27). Patients were simultaneously infused with two 


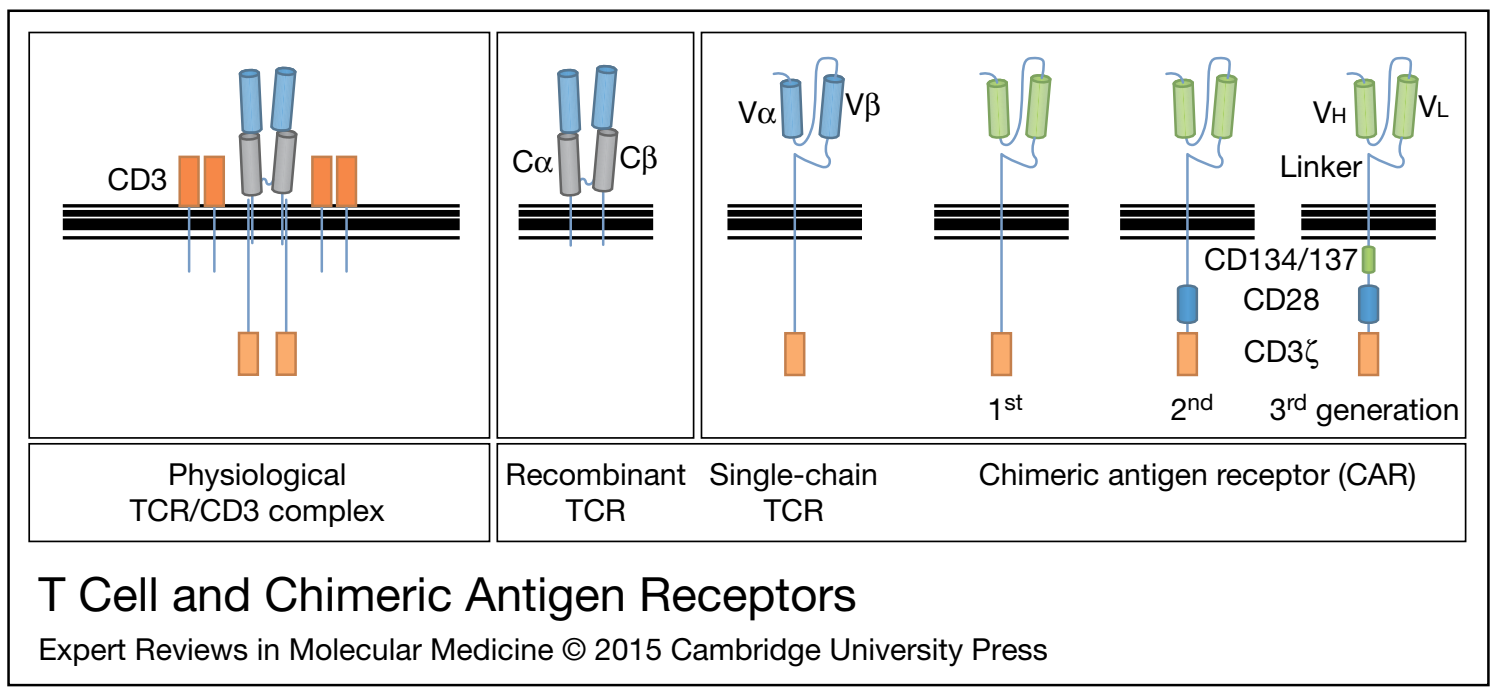

FIGURE 1

T Cell and Chimeric Antigen Receptors. The $\alpha \beta$ T cell receptor (TCR) comprises $\alpha$ - and $\beta$-chains associated with the $\gamma, \delta, \epsilon$ and $\zeta$ chains of the CD3 complex. TCRs recognize specific antigenic peptides that have been processed and presented in association with human leukocyte antigen (HLA) molecules expressed on the surface of target cells. Chimeric antigen receptors (CARs) are recombinant molecules composed of an antigen-specific antibody linked through a hinge region to $\mathrm{CD} 3 \zeta$ alone (first-generation), or in combination with the intracellular signalling domain of a T cell co-stimulatory molecule, usually CD28 (second-generation), or more recently with an additional signalling domain from a second co-stimulatory molecule such as CD137 (4-1BB) or CD134 (third-generation). These receptors mediate high affinity binding of target cell surface proteins and are independent of HLA presentation.

autologous $\mathrm{T}$ cell products expressing CD19 CARs; one CAR encoded both the costimulatory CD28 and the CD3- $\zeta$ domains, while the other encoded only the CD3- $\zeta$ chain. Second-generation CARs drove enhanced expansion and persistence of the modified cells as compared with first-generation CARs. Kochenderfer et al. showed their encouraging results with a patient treated with second-generation chimeric antigen receptor $\mathrm{T}$ cells directed at CD19 with a dramatic tumour regression (Ref. 28). Nonetheless, this patient was treated a second time after relapse alongside seven others, either chronic lymphocytic leukaemia (CLL) or B-cell lymphoma patients. Six of the eight treated patients obtained objective remissions (Ref. 29). Brentjens et.al. enrolled another 10 patients with either chemotherapy refractory CLL or relapsed acute B lymphoblastic leukaemia (B-ALL) on two phase 1 dose escalation clinical trials with second-generation CARs directed at CD19 (Ref. 30). Three of four evaluable patients with bulky CLL exhibited either a significant reduction or a mixed response in peripheral lymphadenopathy. Two recent publications with patients with relapsed or refractory B-ALL who received autologous CD19 CAR modified T cells also demonstrated the marked antitumour efficacy (from 88 up to $100 \%$ ) and reliability of this therapy (Refs 31,32 ). In an allogeneic setting, a clinical trial involving $\mathrm{T}$ cells obtained from each hematopoietic stem cell transplantation (HSCT) donors and genetically modified to express CD19 CARs showed that a relatively small number of these cells can cause regressions of malignancies (CLL and Mantle Cell Lymphoma), which were refractory to other therapies, without causing GvHD (Ref. 33). In another trial (Ref. 34), eight patients with B-cell malignancies who had relapsed after HSCT were infused with donor-derived virusspecific T cells expanded with LCLs and transduced with a $\gamma \mathrm{RV}$ bearing a CD19 CAR transgene. These infusions were safe in all patients, did not induce GvHD, expanded in the presence of Epstein-Barr virus (EBV) reactivation, and in two of six patients could produce objective clinical responses (Ref. 34).

Some subjects have encountered complications following infusion of CAR modified $\mathrm{T}$ cells, with cytokine-mediated toxicities (Ref. 35). A systemic inflammatory syndrome related to cytokine storms mediated by tumor necrosis factor alpha (TNFa) and interleukin-6 (IL-6) has been reported in early phase trials, and measures taken to address such complications include the use of anti-IL-6 antibody therapy (Refs 31, 32).

Overall, $\gamma \mathrm{RV}$ genetic modification of $\mathrm{T}$ cells with CARs established the first proof of concept data for this type of cancer immunotherapy.

\section{HIV therapies}

$\gamma \mathrm{RV}$ vectors have also been used to deliver transgenes in the context of clinical gene therapy for the treatment of HIV. In 1998, a pilot study of three HIV-seropositive patients infused with autologous CD4 cells transduced with a mutant protein that remains one of the most potent inhibitors of HIV replication demonstrated persistence of the Rev-transduced cells for an average of 6 months after retroviral gene transfer (Ref. 36). Although there was no long-term benefit, a modest survival advantage of the transduced CD4 cells was observed. The same year, a phase 1 clinical trial evaluating the safety and effects in asymptomatic 
HIV-1 infected patients of autologous lymphocytes transduced with a ribozyme that cleaves HIV-1 RNA led to similar results (Ref. 37). Studies have also evaluated a receptor comprising transmembrane and extracellular domains of human CD4 and the $\zeta$-subunit of the CD3TCR, to target the gp120 cell surface expressed HIV glycoprotein. A phase II randomised trial showed that infusion of gene-modified, but not unmodified, $\mathrm{T}$ cells was associated with reduced HIV burden in some subjects (Ref. 38) and found that persistence of cells harbouring the chimeric transgene was over 1 year. Deeks' study demonstrated rises in CD4+ T cell levels in patients receiving ex vivo expanded autologous $\mathrm{T}$ cells and modest decreases in viral reservoirs in HIV-infected individuals compared with controls. Similar results were obtained in a study with 24 patients where $\mathrm{CD} 4 \zeta$ was detected in $1-3 \%$ of blood mononuclear cells at 8 weeks and $0.1 \%$ at 1 year after infusion (Ref. 39). These studies confirmed the safety and feasibility of adoptive therapy with $\mathrm{CD} 4 \zeta$ gene-modified $\mathrm{T}$ cells for the treatment of HIV infection. Other more recent trials also involving twins discordant for HIV infection support the hypothesis that anti-HIV genes afford a survival advantage to $\mathrm{T}$ cells and potential benefit to HIV-1 positive individuals (Refs 40, 41). One of the most recent completed clinical trials was tested in ten patients with late-stage HIV infection (Ref. 42). The infusions of transduced $\mathrm{T}$ cells with a $\gamma \mathrm{RV}$ carrying a transgene encoding a HIV entry-inhibitory peptide derived from gp41 were well tolerated, and a significant albeit transient increase of CD4 counts was observed after infusion. T cell gene therapy for HIV might help generate an immune system resistant to HIV and contribute to the control of HIV (Ref. 43). Also, it is likely that such approaches will have to be combined with stem cell modification and ablative conditioning regimens to ensure long-term, thymic and non-thymic mediated $\mathrm{T}$ cell recovery alongside elimination of HIV reservoirs.

\section{Genotoxicity}

One concern associated with $\gamma \mathrm{RVs}$ has been the risk of genotoxicity associated with mutagenic effects elicited by vector insertion near to transcription start sites and transactivation of proto-oncogenes, although in clinical studies, adverse effects have only been reported following hematopoietic stem cell (HSC) modification (Ref. 44). In clinical studies of HSC modification for X-linked SCID-X1 (Refs 45, 46), chronic granulomatous disease (Ref. 47) and Wiskott-Aldrich syndrome (WAS) (Refs 48, 49), leukemic transformation has been reported in Phase 1 trials. In contrast, several hundred patients have now received $\gamma \mathrm{RV}$-modified $\mathrm{T}$ cells without evidence of genotoxicity. Although theoretically possible, it appears that $\mathrm{T}$ cells have a lower risk of transformation than HSCs (Refs 38, 50). However, in vitro and in vivo experiments revealed the susceptibility of primary $\mathrm{T}$ cells to malignant transformation, from a rare event of T-cell immortalisation (Ref. 51) to mature T-cell lymphomas in mice (Ref. 52). Despite integration into genes with potential to mediate mutagenesis, long term studies in patients followed in a clinical trial have demonstrated stable gene expression profiles and phenotype with no evidence of clonal expansion (Ref. 53) presumably because of the differentiated nature of T cells compared with HSCs.

\section{Lentiviral-mediated T cell modification}

In contrast to oncoretroviral preintegration complexes (PICs), lentiviral PIC has the capability of actively transporting into the nucleus through nuclear pore complexes in an adenosine triphosphate (ATP)-dependent manner (Ref. 54). Thus, HIV-1-based LVs have the potential to transduce minimally activated cells and to be pseudotyped with heterologous envelope proteins such as vesicular stomatitis virus $G$ envelope protein, to confer broad tropism for transduction of a wide variety of mammalian cell types including $\mathrm{T}$ cells (Refs 55, 56). First-generation HIV-1 LVs used a split packaging plasmid system and transient transfection to generate virion particles. Second-generation vectors were devoid of viral accessory proteins (Vif, Vpu, Vpr or Nef), deleted of U3-LTR elements (including its thymine-adenine-thymine-adenine (TATA)-box-, Sp1-, nuclear factor kappa B (NF-kB)and nuclear factor of activated T-cells (NFAT)binding sites) and incorporating internal promoter sequences (Refs 57, 58). Third generation LVs are now also Tat-independent with Rev provided in trans by a fourth plasmid (Ref. 59). Additional heterologous elements have improved the properties of the transducing vector, including the woodchuck hepatitis virus post-transcriptional regulatory element used to stabilise transgene mRNA levels and increase transgene expression, and the central polypurine tract, another cis-acting element, is used to improve transduction efficiency (Refs 60, 61). Additional available modifications include the use of chromatin-insulator elements (Ref. 62), chromatin-opening elements (Ref. 63), bioengineered ligand/antibody-displaying envelopes for targeted $\mathrm{T}$ cell transduction (Ref. 64) and tissuespecific promoters (Refs 59, 65). Several envelope glycoproteins can be used to pseudotype LV particles, for example amphotropic murine leukaemia virus (MLV) or RD114 (type D retrovirus) envelope-pseudotyped LVs show efficient transduction of human CD34+ haematopoietic stem cells (Ref. 66), but the vesicular stomatitis virus $\mathrm{G}$ glycoprotein is widely used based on its efficiency and broad tropism. This pseudotype provides resistance to freeze-thawing and sufficient stability for LV to withstand concentration or purification methods (Ref. 67). High concentrations of LV particles are usually needed for efficient gene transfer in primary $\mathrm{T}$ cells, requiring concentration of the viral stock. Ultracentrifugation is commonly employed but also concentrates cellular debris, membrane fragments and proteins derived from producer cells and the culture medium. Such materials can be toxic to target cells, 
in particular to primary cells, and may cause inflammatory or immunogenic reactions for direct in vivo studies (Refs 68, 69).

In the absence of efficient stable packaging systems, most clinical vectors have been produced by transient transfection methods in $293 \mathrm{~T}$ packaging cells, and a number of published clinical studies have now employed this approach including anti-HIV therapy (Ref. 70), adrenoleukodystrophy (ALD) (Ref. 71), $\beta$ Thalassemia (Ref. 72), CLL (Ref. 73), WAS (Ref. 74), and Metachromatic Leukodystrophy (Ref. 75). To date these vectors have a satisfactory safety profile compared with $\gamma$ RVs. Previous mapping of LVs and $\gamma \mathrm{RVs}$ integration sites on the human genome sequence found distinct target site preferences between the two viruses. MLV-based $\gamma$ RVs appear to be biased towards integration near transcription start sites or cytosine-phosphate-guanine $(\mathrm{CpG})$ islands, whereas HIV-based LVs appear to favour integration within active transcription regions. Clonal expansion was reported in a trial for $\beta$ Thalassemia where hematopoietic stem cells were modified with a LV incorporating a modified $\beta$-globin locus control region, and vector integration into the high mobility group AT-hook 2 (HMGA2) locus disrupted endogenous microRNA regulation, but without leukemic transformation (Ref. 72). Of particular note, longitudinal studies of CD4+ T lymphocytes transduced with a LV incorporating anti-HIV envelope sequences have found no evidence of abnormal clonal expansions (Ref. 76).

\section{HIV therapies}

Lentiviral $\mathrm{T}$ cell gene therapies are summarised in Table 1. Levine et al. carried out the first clinical trial in which a conditionally replicating HIV-based LV expressing an antisense gene against the HIV envelope was introduced into T cells of five HIV-infected subjects (Ref. 70). This LV retained full LTRs of HIV with expression of the antisense element upregulated in the presence of wild-type HIV. This LTR-dependent transcriptional upregulation is in contrast to the majority of other trials, where self-inactivating vectors with LTRs modified by deletion of the U3 region, are used to reduce the risk of vector mobilisation. After 1 year, all patients showed a decrease of the viral load and four out of five showed an increase in the number of CD4+ $\mathrm{T}$ cells. The transduced cells diminished in number after infusion but were detectable at 2 years in three of five subjects (Ref. 70). A follow-on Phase I/II clinical trial was initiated to evaluate the distribution of integrated vector copies in transduced CD4+ $\mathrm{T}$ cells before and after reinfusion with no evidence of vector-mediated transactivation, which had caused clonal expansions in some stem cell studies (Ref. 76).

\section{CAR gene therapies}

The groundbreaking studies on CAR came from June lab in 2011 with CD19 CARs carried by LVs (Refs 73, 77, 78). They tested the feasibility and safety of CAR CD19-specific in patients with CD19positive lymphoid malignancies, including refractory CLL (Ref. 73) and acute lymphoblastic leukaemia (ALL) (Ref. 77). The LV expressed a second-generation CAR with specificity for the B-cell antigen CD19, coupled with 4-1BB, a costimulatory receptor in $T$ cells, and CD3-zeta signalling domains. Interestingly, in the CLL setting relatively low dose anti-CD19 CAR cells underwent $>1000$-fold in vivo expansion. These cells trafficked to tumour sites, persisted longterm in vivo and induced rapid and potent antitumour activity in the chemotherapy refractory CLL patient. No clonal proliferation of the infused $\mathrm{T}$ cells was observed, although two toxic effects were described, an unexpected occurrence of delayed tumour lysis syndrome and lymphopenia. Remission was on-going 10 months after treatment (Ref. 73). The two ALL patients developed a severe cytokine-release syndrome, and a single course of anti-cytokine therapy, consisting of a TNF inhibitor and anti-IL6 receptor monoclonal antibody, was effective in reversing the syndrome and did not prevent expansion of CAR T cells or reduce antileukemic efficacy (Ref. 77). Cells persisted as memory $\mathrm{CAR}+\mathrm{T}$ cells and retained anti-CD19 effector functionality, indicating the potential of this MHCindependent approach for the effective treatment of B cell malignancies (Ref. 78).

\section{$\alpha \beta$ TCR gene therapies}

In a recent trial carried out with an affinity-enhanced TCR specific for the HLA-A*01-restricted MAGEA3 tumour antigen, a melanoma and a myeloma patient were treated with autologous modified T cells. Both subjects encountered severe cardiac toxicity owing to off-target reactivity, developed cardiogenic shock and died within a few days of T-cell infusion (Refs 79, 80). This was an unexpected and unpredictable consequence of cross recognition of unrelated cardiac antigen by the introduced TCR, underlining the difficulty in modelling such consequences ahead of clinical application.

Additional studies that are planned include treatment of patients with locally advanced or metastatic melanoma, with $\mathrm{CD} 8+\mathrm{T}$ cells engineered to express the $\alpha \beta$ chains of a high affinity TCR specific for the HLA-A*0201-restricted MART-1 melanoma tumour antigen, together with the HSVl-sr39tk suicide gene, or with autologous PBLs expressing a $\alpha \beta$ TCR specific for tyrosinase. For myeloma, MAGE-A3 and NYESO-1 TCRs post-ASCT and for the treatment of synovial sarcoma, NY-ESO-1 TCR transgenes will also be carried by LVs, as NY-ESO-1 and LAGE-1 TCRs for hepatocellular carcinomas.

For HIV therapy, gag-specific TCR (the University of Pennsylvania, Philadelphia) and RNAi (Beckman Research Institute) are expressed in autologous $\mathrm{T}$ cells and target HIV Tat and Rev. Calimmune developed a dual therapeutic anti-HIV LV that down-regulates CCR5 and inhibits HIV-1 fusion via cell surface 
expression of the gp41-derived C46 peptide, initially developed by D. von Laer's group (Ref. 81). This shRNA construct is being tested in Phase I/II trials by engineering HIV-resistant hematopoietic cells and CD4+ T cells (Ref. 82).

For ovarian cancer, an alpha-folate CAR, following a clinical trial with $\gamma \mathrm{RV}$ (Ref. 24), and a mesothelin CAR are tested in clinical trials currently recruiting from the University of Pennsylvania, Philadelphia. In nonHodgkin lymphoma, CD19 CAR only, CD19 CAR together with a truncated EGFR in central memory $\mathrm{T}$ cells, or bi-specific CMV-TCR and CD19 CAR T cells will be infused to patients. For acute lymphoid leukaemia, CD19 CARs have also been designed in an autologous setting. Finally, patients with B-lymphoid malignancies will be infused with allogeneic CD8T cells expressing CD19 CARs (data collected from http://www.wiley.com/legacy/wileychi/ genmed/clinical/ and http://www.clinicaltrials.gov/). Interestingly, several companies including big pharmaceuticals have taken over to develop CAR T cells into clinical products (Ref. 83).

\section{Non-viral transposon-based T cell engineering}

Non-viral gene transfer technologies offer the prospect of more cost effective and less biologically complex alternatives to viral methodologies, with reduced concerns related to safety, host immune response and manufacturing costs. Plasmid-based gene transfer could facilitate investigation of a greater variety of transgene cassettes, and allow rapid evaluation of new receptors. This is an important consideration in the context of $\alpha \beta$ TCRs, where multiple HLA-peptide specific receptors will need to be provided, and for CAR receptors, which continue to rapidly evolve with revised costimulatory and selection domains.

DNA transposons have the ability to insert transgenes into host chromosomes for long-term transgene expression and are derived from natural genetic elements residing in the genome as repetitive sequences that transfer through a direct cut-and-paste mechanism, called transposition. Sleeping Beauty, a reactivated $\mathrm{Tc} 1 /$ mariner-like element from fossils of the salmonid fish genome (Ref. 84), Medaka fish Tol2, a member of the hAT family (Ref. 85) and cabbage looper moth piggybac (Ref. 86), the founding member of the piggyBac family, are all transposons that effectively transpose in mammalian cells (Ref. 87). Advantages in using these systems for genetic modification of $\mathrm{T}$ cells include reduced costs associated with the manufacturing of clinical-grade plasmid-based vectors, compared with recombinant viral vectors, and reduced immunogenicity. In addition, transposon vectors may tolerate larger and more complex transgenes. Unlike LTRs of retroviruses, the terminal inverted repeats of SB vectors have very low intrinsic enhancer/promoter activity and cannot readily activate endogenous genes that flank the transposon integration sites. A reconstruction based on several rounds of mutagenesis established a functional SB transposase protein that was found to efficiently catalyse transposition in human cells (Ref. 84). Mutagenesis of the transposase gene led to a higher efficiency variant (Ref. 88) with the development of the SB100X transposase (Ref. 89).

In primary human $\mathrm{T}$ cells (Table 1), Huang et al. demonstrated that SB transposons can mediate stable transposition of reporter genes with high levels of transgene expression maintained at least 4 months in vitro (Ref. 90). Transposon constructs expressing tumour antigen-specific TCR genes targeting p53 and MART1 also showed sustained expression and furthermore, functional reactivity of transposon-engineered lymphocytes on encountering target antigen presented on tumour cells. A direct comparison between transposonand retroviral-modified lymphocytes revealed a comparable transgene expression and phenotypic function (Ref. 91). Comparison of lentiviral- and sleeping beauty-mediated $\alpha \beta$ TCR gene transfer supported the exploitation of the SB plasmid based system as a flexible and adaptable platform for accelerated, early-phase assessment of TCR gene therapies (Ref. 92). SB delivery of CD19 CARs has led to early-phase human trials that are currently assessing safety and feasibility of administering these autologous genetically modified $\mathrm{T}$ cells into patients with high-risk B-lymphoid malignancies undergoing autologous HSCT. T cells with redirected specificity were efficiently generated with plasmids from the Sleeping Beauty system, and expansion was achieved for both $\mathrm{CD} 4+$ and $\mathrm{CD} 8+\mathrm{T}$ cells expressing CAR with CD19+ artificial antigen-presenting cells (Refs 93, 94).

Overall, eight clinical trials have opened recently using the Sleeping Beauty system for non-viral ex vivo genetic modification of either autologous $T$ cells, HLA-matched allogeneic $\mathrm{T}$ lymphocytes or umbilical cord blood lymphocytes for the treatment of lymphoma, and autologous $\mathrm{T}$ cells for the treatment of CLL at the Anderson Cancer Center and the University of Texas (http://www.wiley.com/legacy/ wileychi/genmed/clinical/), with no acute toxicities reported to date.

\section{Future developments}

The rapid advancement of genome editing techniques holds much promise for the field of human gene therapy. Among the upcoming methods of lymphocyte genetic modification, the zinc finger nuclease (ZFN) technology (Refs 95, 96) is currently being evaluated in early stage clinical trials infusing HIV-resistant $T$ cells generated by ZFN-mediated disruption of the CCR5 coreceptor for HIV-I (Ref. 97). A similar concept aims to engineer $\mathrm{T}$ cells to express TCR or CAR after disruption of endogenous TCR by designer ZFNs or TALE nucleases (TALENs) is being investigated. These approaches aim to eliminate alloreactivity by knocking out $\alpha \beta$ TCR expression and are combined with viral or SB transfer of antigen specific receptors. 
Thus, it may be possible to generate universal allogeneic tumour-associated antigen-specific $\mathrm{T}$ cells from one donor that might be administered to multiple patients as off-the-shelf $\mathrm{T}$ cell gene therapies (Ref. 98). Sangamo have developed ZFNs to target the $\alpha$ and $\beta$ chain constant regions (Refs 98, 99), and Cellectis, TALENs (Abstract ESGT, A45, Or035, http://online. liebertpub.com/doi/pdfplus/10.1089/hum.2013.

2513). A recent publication describes TALENs efficient genome editing in primary $\mathrm{T}$ lymphocytes (Ref. 100). Expression of these reagents from transfected mRNA is being investigated and similar approach for the transient expression of $\alpha \beta$ specific receptors following electroporation of mRNA is being investigated as an alternative to stable transduction. In some circumstances, time limited expression of antigen specific receptors may be desirable, allowing clinical effects with reduced risk of sustained adverse effects (Ref. 101).

Clustered regularly interspaced short palindromic repeats (CRISPR) are clusters of brief DNA sequences that read similarly forward and backward, found in many types of bacteria. Since 2013, the CRISPR/Cas system has been used for gene editing (adding, disrupting or changing the sequence of specific genes) and gene regulation in various species. By delivering the Cas9 protein and appropriate guide RNAs into a cell, the genome can be cut at any desired location (Ref. 102). Therefore, CRISPR could make gene therapies more broadly applicable, from simple genetic disorders to eventually more complex diseases involving multiple genes (Ref. 103).

Overall, engineered $T$ cells offer enormous therapeutic potential, with technologies evolving to allow modification of either autologous or allogeneic populations, which will need to be carefully assessed in welldesigned clinical studies.

\section{Financial support}

W. Q receives support from Great Ormond Street Hospital Special Trustees and the National Institute of Health Research via Biomedical research councils. A. C. F was supported by Children with Cancer UK.

\section{Conflict of Interest}

Cellectis, Calimmune, and Miltenyi Biotec provide research funding to UCL and are collaborating in the development of $\mathrm{T}$ cell therapies, but have had no role in the writing or review of this manuscript.

\section{References}

1. Ginn S.L. et al. (2013) Gene therapy clinical trials worldwide to 2012 - an update. Journal of Gene Medicine 15, 65-77

2. Rosenberg S.A. et al. (1990) Gene transfer into humans-immunotherapy of patients with advanced melanoma, using tumor-infiltrating lymphocytes modified by retroviral gene transduction. New England Journal of Medicine 323, 570-578

3. Blaese R.M. et al. (1995) T lymphocyte-directed gene therapy for ADA-SCID: initial trial results after 4 years. Science $\mathbf{2 7 0}$, $475-480$
4. Bordignon C. et al. (1995) Gene therapy in peripheral blood lymphocytes and bone marrow for ADA- immunodeficient patients. Science 270, 470-475

5. Muul L.M. et al. (2003) Persistence and expression of the adenosine deaminase gene for 12 years and immune reaction to gene transfer components: long-term results of the first clinical gene therapy trial. Blood 101, 2563-2569

6. Riddell S.R. et al. (1996) T-cell mediated rejection of genemodified HIV-specific cytotoxic T lymphocytes in HIVinfected patients. Nature Medicine 2, 216-223

7. Bonini C. et al. (1997) HSV-TK gene transfer into donor lymphocytes for control of allogeneic graft-versus-leukemia. Science 276, 1719-1724

8. Burt R.K. et al. (2003) Herpes simplex thymidine kinase genetransduced donor lymphocyte infusions. Experimental Hematology 31, 903-910

9. Tiberghien P. et al. (2001) Administration of herpes simplexthymidine kinase-expressing donor $\mathrm{T}$ cells with a T-celldepleted allogeneic marrow graft. Blood 97, 63-72

10. Fehse B. et al. (2004) Evidence for increased risk of secondary graft failure after in vivo depletion of suicide gene-modified $\mathrm{T}$ lymphocytes transplanted in conjunction with CD34+ enriched blood stem cells. Blood 104, 3408-3409

11. Ciceri F. et al. (2009) Infusion of suicide-gene-engineered donor lymphocytes after family haploidentical haemopoietic stem-cell transplantation for leukaemia (the TK007 trial): a non-randomised phase I-II study. Lancet Oncology 10, 489-500

12. Vago L. et al. (2012) T-cell suicide gene therapy prompts thymic renewal in adults after hematopoietic stem cell transplantation. Blood 120, 1820-1830

13. Borchers S. et al. (2011) Genetically modified donor leukocyte transfusion and graft-versus-leukemia effect after allogeneic stem cell transplantation. Human Gene Therapy 22, 829-841

14. Zhan H. et al. (2013) Production and first-in-man use of T cells engineered to express a HSVTK-CD34 sort-suicide gene. PLoS ONE 8, e77106

15. Di Stasi A. et al. (2011) Inducible apoptosis as a safety switch for adoptive cell therapy. New England Journal of Medicine 365, 1673-1683

16. Morgan R.A. et al. (2006) Cancer regression in patients after transfer of genetically engineered lymphocytes. Science $\mathbf{3 1 4}$, 126-129

17. Burns W.R. et al. (2009) Lack of specific gamma-retroviral vector long terminal repeat promoter silencing in patients receiving genetically engineered lymphocytes and activation upon lymphocyte restimulation. Blood 114, 2888-2899

18. Johnson L.A. et al. (2009) Gene therapy with human and mouse T-cell receptors mediates cancer regression and targets normal tissues expressing cognate antigen. Blood 114, 535-546

19. Robbins P.F. et al. (2011) Tumor regression in patients with metastatic synovial cell sarcoma and melanoma using genetically engineered lymphocytes reactive with NY-ESO-1. Journal of Clinical Oncology 29, 917-924

20. Morgan R.A. et al. (2013) Cancer regression and neurological toxicity following anti-MAGE-A3 TCR gene therapy. Journal of Immunotherapy 36, 133-151

21. Qasim W. et al. (2013) Production and use of autologous gene modified $\mathrm{T}$ cells targeting metastatic hepatitis B associated hepatocellular carcinoma. Human Gene Therapy A25-A26

22. Sadelain M., Brentjens R. and Riviere I. (2013) The basic principles of chimeric antigen receptor design. Cancer Discovery 3, 388-398

23. Lamers C.H. et al. (2006) Process validation and clinical evaluation of a protocol to generate gene-modified T lymphocytes for imunogene therapy for metastatic renal cell carcinoma: GMP-controlled transduction and expansion of patient's T lymphocytes using a carboxy anhydrase IX-specific scFv transgene. Cytotherapy 8, 542-553

24. Kershaw M.H. et al. (2006) A phase I study on adoptive immunotherapy using gene-modified $\mathrm{T}$ cells for ovarian cancer. Clinical Cancer Research 12, 6106-6115

25. Pule M.A. et al. (2008) Virus-specific T cells engineered to coexpress tumor-specific receptors: persistence and antitumor activity in individuals with neuroblastoma. Nature Medicine 14, 1264-1270 
26. Louis C.U. et al. (2011) Antitumor activity and long-term fate of chimeric antigen receptor-positive $\mathrm{T}$ cells in patients with neuroblastoma. Blood 118, 6050-6056

27. Savoldo B. et al. (2011) CD28 costimulation improves expansion and persistence of chimeric antigen receptor-modified $\mathrm{T}$ cells in lymphoma patients. Journal of Clinical Investigation 121, 1822-1826

28. Kochenderfer J.N. et al. (2010) Eradication of B-lineage cells and regression of lymphoma in a patient treated with autologous T cells genetically engineered to recognize CD19. Blood 116, 4099-4102

29. Kochenderfer J.N. et al. (2012) B-cell depletion and remissions of malignancy along with cytokine-associated toxicity in a clinical trial of anti-CD19 chimeric-antigen-receptortransduced T cells. Blood 119, 2709-2720

30. Brentjens R.J. et al. (2011) Safety and persistence of adoptively transferred autologous CD19-targeted T cells in patients with relapsed or chemotherapy refractory B-cell leukemias. Blood 118, 4817-4828

31. Brentjens R.J. et al. (2013) CD19-targeted T cells rapidly induce molecular remissions in adults with chemotherapyrefractory acute lymphoblastic leukemia. Science Translational Medicine 5, 177ra38

32. Davila M.L. et al. (2014) Efficacy and toxicity management of 19-28z CAR T cell therapy in B cell acute lymphoblastic leukemia. Science Translational Medicine 6, $224 \mathrm{ra} 25$

33. Kochenderfer J.N. et al. (2013) Donor-derived CD19-targeted $T$ cells cause regression of malignancy persisting after allogeneic hematopoietic stem cell transplantation. Blood 122, 41294139

34. Cruz C.R. et al. (2013) Infusion of donor-derived CD19-redirected virus-specific $\mathrm{T}$ cells for B-cell malignancies relapsed after allogeneic stem cell transplant: a phase 1 study. Blood 122, 2965-2973

35. Morgan R.A. et al. (2010) Case report of a serious adverse event following the administration of $\mathrm{T}$ cells transduced with a chimeric antigen receptor recognizing ERBB2. Molecular Therapy 18, 843-851

36. Ranga U. et al. (1998) Enhanced T cell engraftment after retroviral delivery of an antiviral gene in HIV-infected individuals. Proceedings of the National Academy of Sciences of the United States of America 95, 1201-1206

37. Wong-Staal F., Poeschla E.M. and Looney D.J. (1998) A controlled, Phase 1 clinical trial to evaluate the safety and effects in HIV-1 infected humans of autologous lymphocytes transduced with a ribozyme that cleaves HIV-1 RNA. Human Gene Therapy 9, 2407-2425

38. Deeks S.G. et al. (2002) A phase II randomized study of HIVspecific T-cell gene therapy in subjects with undetectable plasma viremia on combination antiretroviral therapy. Molecular Therapy 5, 788-797

39. Mitsuyasu R.T. et al. (2000) Prolonged survival and tissue trafficking following adoptive transfer of CD4zeta genemodified autologous $\mathrm{CD} 4(+)$ and $\mathrm{CD} 8(+) \mathrm{T}$ cells in human immunodeficiency virus-infected subjects. Blood 96, 785-793

40. Macpherson J.L. et al. (2005) Long-term survival and concomitant gene expression of ribozyme-transduced CD4+ Tlymphocytes in HIV-infected patients. Journal of Gene Medicine 7, 552-564

41. Morgan R.A. et al. (2005) Preferential survival of CD4+ T lymphocytes engineered with anti-human immunodeficiency virus (HIV) genes in HIV-infected individuals. Human Gene Therapy 16, 1065-1074

42. van Lunzen J. et al. (2007) Transfer of autologous gene-modified $\mathrm{T}$ cells in HIV-infected patients with advanced immunodeficiency and drug-resistant virus. Molecular Therapy 15, 1024-1033

43. Hoxie J.A. and June C.H. (2012) Novel cell and gene therapies. Cold Spring Harbor Perspectives in Medicine 2(10). pii: a007179. doi: 10.1101/cshperspect.a007179.

44. Wu X. et al. (2003) Transcription start regions in the human genome are favored targets for MLV integration. Science 300, 1749-1751

45. Hacein-Bey-Abina S. et al. (2003) LMO2-associated clonal T cell proliferation in two patients after gene therapy for SCIDX1. Science 302, 415-419
46. Hacein-Bey-Abina S. et al. (2008) Insertional oncogenesis in 4 patients after retrovirus-mediated gene therapy of SCID-X1. Journal of Clinical Investigation 118, 3132-3142

47. Ott M.G. et al. (2006) Correction of X-linked chronic granulomatous disease by gene therapy, augmented by insertional activation of MDS1-EVI1, PRDM16 or SETBP1. Nature Medicine 12, 401-409

48. Boztug K. et al. (2010) Stem-cell gene therapy for the Wiskott-Aldrich syndrome. New England Journal of Medicine 363, 1918-1927

49. Braun C.J. et al. (2014) Gene therapy for wiskott-Aldrich syndrome-long-term efficacy and genotoxicity. Science Translational Medicine 6, 227ra33

50. Newrzela S. et al. (2008) Resistance of mature T cells to oncogene transformation. Blood 112, 2278-2286

51. Newrzela S. et al. (2011) Retroviral insertional mutagenesis can contribute to immortalization of mature $\mathrm{T}$ lymphocytes. Molecular Medicine 17, 1223-1232

52. Heinrich T. et al. (2013) Mature T-cell lymphomagenesis induced by retroviral insertional activation of Janus kinase 1 . Molecular Therapy 21, 1160-1168

53. Recchia A. et al. (2006) Retroviral vector integration deregulates gene expression but has no consequence on the biology and function of transplanted T cells. Proceedings of the National Academy of Sciences of the United States of America 103, 1457-1462

54. Bukrinsky M. (2004) A hard way to the nucleus. Molecular Medicine 10, 1-5

55. Bukrinsky M.I. et al. (1993) A nuclear localization signal within HIV-1 matrix protein that governs infection of non-dividing cells. Nature 365, 666-669

56. Naldini L. et al. (1996) In vivo gene delivery and stable transduction of nondividing cells by a lentiviral vector. Science 272, 263-267

57. Miyoshi H. et al. (1998) Development of a self-inactivating lentivirus vector. Journal of Virology 72, 8150-8157

58. Zufferey R. et al. (1998) Self-inactivating lentivirus vector for safe and efficient in vivo gene delivery. Journal of Virology 72, 9873-9880

59. Sakuma T., Barry M.A. and Ikeda Y. (2012) Lentiviral vectors: basic to translational. Biochemical Journal 443, 603-618

60. Dupuy F.P. et al. (2005) Lentiviral transduction of human hematopoietic cells by HIV-1- and SIV-based vectors containing a bicistronic cassette driven by various internal promoters. Journal of Gene Medicine 7, 1158-1171

61. Zufferey R. et al. (1999) Woodchuck hepatitis virus posttranscriptional regulatory element enhances expression of transgenes delivered by retroviral vectors. Journal of Virology $\mathbf{7 3}$, 2886-2892

62. Osti D. et al. (2006) Comparative analysis of molecular strategies attenuating positional effects in lentiviral vectors carrying multiple genes. Journal of Virological Methods 136, 93-101

63. Zhang F. et al. (2007) Lentiviral vectors containing an enhancer-less ubiquitously acting chromatin opening element (UCOE) provide highly reproducible and stable transgene expression in hematopoietic cells. Blood $\mathbf{1 1 0}$, 1448-1457

64. Funke S. et al. (2008) Targeted cell entry of lentiviral vectors. Molecular Therapy 16, 1427-1436

65. Dropulic B. (2011) Lentiviral vectors: their molecular design, safety, and use in laboratory and preclinical research. Human Gene Therapy 22, 649-657

66. Relander T. et al. (2005) Gene transfer to repopulating human CD34+ cells using amphotropic-, GALV-, or RD114-pseudotyped HIV-1-based vectors from stable producer cells. Molecular Therapy 11, 452-459

67. Kutner R.H., Zhang X.Y. and Reiser J. (2009) Production, concentration and titration of pseudotyped HIV-1-based lentiviral vectors. Nature Protocols 4, 495-505

68. Baekelandt V. et al. (2003) Optimized lentiviral vector production and purification procedure prevents immune response after transduction of mouse brain. Gene Therapy 10, 19331940

69. Tuschong L. et al. (2002) Immune response to fetal calf serum by two adenosine deaminase-deficient patients after $\mathrm{T}$ cell gene therapy. Human Gene Therapy 13, 1605-1610 
70. Levine B.L. et al. (2006) Gene transfer in humans using a conditionally replicating lentiviral vector. Proceedings of the National Academy of Sciences of the United States of America 103, 17372-17377

71. Cartier N. et al. (2009) Hematopoietic stem cell gene therapy with a lentiviral vector in X-linked adrenoleukodystrophy. Science 326, 818-823

72. Cavazzana-Calvo M. et al. (2010) Transfusion independence and HMGA2 activation after gene therapy of human beta-thalassaemia. Nature 467, 318-322

73. Porter D.L. et al. (2011) Chimeric antigen receptor-modified T cells in chronic lymphoid leukemia. New England Journal of Medicine 365, 725-733

74. Aiuti A. et al. (2013) Lentiviral hematopoietic stem cell gene therapy in patients with Wiskott-Aldrich syndrome. Science 341, 1233151

75. Biffi A. et al. (2013) Lentiviral hematopoietic stem cell gene therapy benefits metachromatic leukodystrophy. Science $\mathbf{3 4 1}$, 1233158

76. Wang G.P. et al. (2009) Analysis of lentiviral vector integration in HIV + study subjects receiving autologous infusions of gene modified CD4+ T cells. Molecular Therapy 17, 844-850

77. Grupp S.A. et al. (2013) Chimeric antigen receptor-modified T cells for acute lymphoid leukemia. New England Journal of Medicine 368, 1509-1518

78. Kalos M. et al. (2011) T cells with chimeric antigen receptors have potent antitumor effects and can establish memory in patients with advanced leukemia. Science Translational Medicine 3, 95ra73

79. Linette G.P. et al. (2013) Cardiovascular toxicity and titin cross-reactivity of affinity-enhanced $\mathrm{T}$ cells in myeloma and melanoma. Blood 122, 863-871

80. Cameron B.J. et al. (2013) Identification of a Titin-derived HLA-A1-presented peptide as a cross-reactive target for engineered MAGE A3-directed T cells. Science Translational Medicine 5, 197ra103

81. Hildinger M. et al. (2001) Membrane-anchored peptide inhibits human immunodeficiency virus entry. Journal of Virology 75, 3038-3042

82. Burke B.P. et al. (2014) CCR5 as a natural and modulated target for inhibition of HIV. Viruses 6, 54-68

83. Flemming A. (2014) Deal watch: Pfizer and GSK join race for T cell cancer therapies. Nature Review Drug Discovery 13, 568-569

84. Ivics Z. et al. (1997) Molecular reconstruction of Sleeping Beauty, a Tc1-like transposon from fish, and its transposition in human cells. Cell 91, 501-510

85. Kawakami K. (2007) Tol2: a versatile gene transfer vector in vertebrates. Genome Biology 8(Suppl 1), S7

86. Ding S. et al. (2005) Efficient transposition of the piggyBac (PB) transposon in mammalian cells and mice. Cell 122, 473-483

87. Hackett P.B., Largaespada D.A. and Cooper L.J. (2010) A transposon and transposase system for human application. Molecular Therapy 18, 674-683

88. Yant S.R et al. (2004) Mutational analysis of the N-terminal DNA-binding domain of sleeping beauty transposase: critical residues for DNA binding and hyperactivity in mammalian cells. Molecular and Cellular Biology 24, 9239-9247

89. Mates L. et al. (2009) Molecular evolution of a novel hyperactive Sleeping Beauty transposase enables robust stable gene transfer in vertebrates. Nature Genetics 41, 753-761

90. Huang X. et al. (2006) Stable gene transfer and expression in human primary $\mathrm{T}$ cells by the Sleeping Beauty transposon system. Blood 107, 483-491

91. Peng P.D. et al. (2009) Efficient nonviral Sleeping Beauty transposon-based TCR gene transfer to peripheral blood lymphocytes confers antigen-specific antitumor reactivity. Gene Therapy 16, 1042-1049

92. Field A.C. et al. (2013) Comparison of lentiviral and sleeping beauty mediated alphabeta $\mathrm{T}$ cell receptor gene transfer. PLoS ONE 8, e68201

93. Kebriaei P. et al. (2012) Infusing CD19-directed T cells to augment disease control in patients undergoing autologous hematopoietic stem-cell transplantation for advanced Blymphoid malignancies. Human Gene Therapy 23, 444-450

94. Singh H. et al. (2014) A new approach to gene therapy using Sleeping Beauty to genetically modify clinical-grade T cells to target CD19. Immunological Reviews 257, 181-190

95. Perez E.E. et al. (2008) Establishment of HIV-1 resistance in CD4+ T cells by genome editing using zinc-finger nucleases. Nature Biotechnology 26, 808-816

96. Maier D.A. et al. (2013) Efficient clinical scale gene modification via zinc finger nuclease-targeted disruption of the HIV co-receptor CCR5. Human Gene Therapy 24, 245-258

97. Tebas P. et al. (2014) Gene editing of CCR5 in autologous CD4T cells of persons infected with HIV. New England Journal of Medicine 370, 901-910

98. Torikai H. et al. (2012) A foundation for universal T-cell based immunotherapy: $\mathrm{T}$ cells engineered to express a CD19-specific chimeric-antigen-receptor and eliminate expression of endogenous TCR. Blood 119, 5697-5705

99. Provasi E. et al. (2012) Editing T cell specificity towards leukemia by zinc finger nucleases and lentiviral gene transfer. Nature Medicine 18, 807-815

100. Berdien B. et al. (2014) TALEN-mediated editing of endogenous T-cell receptors facilitates efficient reprogramming of $\mathrm{T}$ lymphocytes by lentiviral gene transfer. Gene Therapy $\mathbf{2 1}$ 539-548

101. Koh S. et al. (2013) A practical approach to immunotherapy of hepatocellular carcinoma using $\mathrm{T}$ cells redirected against hepatitis B virus. Molecular Therapy-Nucleic Acids 2, e114

102. Mali P., Esvelt K.M. and Church G.M. (2013) Cas9 as a versatile tool for engineering biology. Nature Methods 10, 957 963

103. Meissner T.B. et al. (2014) Genome editing for human gene therapy. Methods in Enzymology 546, 273-295

*Corresponding author:

Anne-Christine Field,

Molecular immunology Unit,

Institute of Child Health (ICH),

University College London (UCL),

30 Guilford Street,

London WC1N 1EH, UK

E-mail: anne-christine.field@ucl.ac.uk 